\title{
Navigating through the gray (and CMYK) areas of figure manipulation: rules at the $\mathrm{JCl}$
}

\begin{abstract}
Since it seems many of the articles we're accepting continue to have image rearrangements and changes in them that are not consistent with JCI policy, we thought it might be a good idea to spell out exactly what is, and more importantly what isn't, allowed.
\end{abstract}

Members of the editorial board at the University of Pennsylvania have now had about 6 months to get properly settled in as editors, reading papers with an editor's scrutiny. And more often than we would like, we are finding a need to scratch our heads regarding how primary image data have been massaged to prepare figures. We've run into several examples in these last six months that have prompted long discussions about what is and is not permitted in terms of image presentation and manipulation. Thus, we decided it was time to be explicit and to establish some new rules going forward.

Most of the examples have been related to gels - blots of all types: Western, Northern, Southern, and PCR. We've commented in the past that it is not acceptable to reuse bands previously published or those that have been rotated in any way; this amounts to fraudulent data manipulation. While that much is clear, a grayer area relates to rearranging lanes; it has long been accepted that an author can splice noncontiguous lanes together in a presented figure, but it has always been our assumption that this is only allowed when the lanes were run on the same gel at the same time. We emphasize this because we have been surprised that a small but not insignificant minority does not share this approach. So we highlight again what the vast majority of our authors already know: you cannot mix and match bands from various gels that were run at different times or exposed for different amounts of time and pass the aggregate off as data that appear to have been run simultaneously and generated under identical conditions.
Going forward, we have adopted the rules established by the Journal of Cell Biology: if you are going to splice lanes together that are noncontiguous, you must insert a thin black or white line between the lanes and explicitly mention this in the figure legend (1). Consistent with these rules, we also repeat an earlier published message: do not remove background noise or doublet bands (2). Even if you think the data are unaffected by removing this information, selective editing or changing of specific features is data manipulation and undermines confidence in your results. It is perfectly acceptable to present blots that didn't run straight or those with some background noise - those are the data we know to trust. We all realize that "representative" usually means best. And the best way to convince us of the data is to quantify the band intensity of repeated blots - statistical significance does not lie.

Regarding histology and, specifically, fluorescent cells on a black background, we borrow again from the JCB. If you are going to make a composite of several cells from adjacent fields, you must draw a border that indicates the cells were not in the same field and make a note to this effect in the legend. We recently made an investigator repeat all the experiments described in a paper because the figures (largely fluorescent histology) had much of the background information removed in order to enhance the visual areas of interest. Areas of overexposure and high intensity were erased, as the investigators thought they would detract from the overall message of the figure. Editors and readers alike are able to see past background noise and aberrant staining if the data merit.

All digital images are subjected to detailed analysis by our production team, who are skilled and experienced in the ways of Photoshop. If we detect any of the alterations noted above, we will then ask for your primary data, and this will needlessly delay publication of your article. If, in fact, we believe that the manipulations change the interpretation of the primary data, we reserve the right to revoke acceptance. As stated before, we work with research integrity officers at various institutions when an investigation into data quality is necessary (2).

We suspect we are mostly preaching to the converted. We are certain that the overwhelming majority of scientists strongly agree with the importance of data integrity and would never think of deliberately manipulating data to mislead the reader. While some of the actions prohibited above may not constitute actual fraud, they do give the appearance of potentially fraudulent intent and can throw a cloud of suspicion over the entire paper. All scientists understand that figures aren't always as beautiful as they might like, and readers, reviewers, and editors are very tolerant of this. They are far less forgiving if they feel they were misled. If the science is beautiful, then the picture doesn't need a makeup artist.

\section{Ushma Neill Executive Editor}

\section{Laurence A. Turka Editor in Chief}

1. Rosser, M. and Yamada, K.M. 2004. What's in a picture? The temptation of image manipulation. J. Cell Biol. 166:11-15.

2. Neill, U.S. 2006. Stop misbehaving! J. Clin. Invest. 116:1740-1741. doi:10.1172/JCI28824 